\title{
Superconductor Hybrids-Electronic Paths to Quantum Computing
}

\author{
Henning Soller \\ Institut für Theoretische Physik, Universität Heidelberg, Heidelberg, Germany \\ Email: hsoller@googlemail.com
}

How to cite this paper: Soller, H. (2017) Superconductor Hybrids-Electronic Paths to Quantum Computing. Journal of Applied Mathematics and Physics, 5, 606-622. https://doi.org/10.4236/jamp.2017.53052

Received: February 5, 2017

Accepted: March 7, 2017

Published: March 10, 2017

Copyright $\odot 2017$ by author and Scientific Research Publishing Inc. This work is licensed under the Creative Commons Attribution International License (CC BY 4.0).

http://creativecommons.org/licenses/by/4.0/

\begin{abstract}
We review several recent theoretical and experimental results in the study of superconductor hybrids. This includes the recent experimental advances in the study of superconducting beamsplitters as well as more advanced superconductor hybrid systems including ferromagnets or Majorana fermions. In the same manner, theoretical studies have revealed that such superconductor hybrid systems pave the way towards electronic generation and detection of entanglement as well as possible use cases in quantum computing. We will review the aspects in detail and illustrate the possible next steps to be taken.
\end{abstract}

\section{Keywords}

Superconductor Hybrids, Superconducting Beamsplitter, Full Counting Statistics, Nanoelectronics, Superconductors

\section{Introduction}

Since their first observation in 1911 [1] superconductors have been the focus of interest of many theoretical and experimental studies. The interest became even greater when it was realized that superconductivity can be connected to a new ground state of the material with all electrons within an energy gap $\pm \Delta$ around the Fermi energy condensing into Cooper pairs. In spite of interesting aspects of exotic and high $T_{c}$ superconductors, we will confine ourselves to classical superconductors described by the Bardeen-Cooper-Schrieffer theory [2] in the following. In this case, the ground state of the system becomes a Cooper pair of two electrons forming a spin-singlet state. The investigation of this new ground state triggered the whole investigation of superconductivity and its specific implications [3].

Moving to nanoelectronics, superconductors have regained interest experimentally and theoretically [4] due to the fact that on a nanoscale superconductors can be effectively connected to other materials forming hybrids [5] [6] and 
can be confined to one-dimensional structures [7]. These possibilities allow for hybrid nanoelectronic superconducting devices that have been studied in a number of ways.

The first immediate observation is that as soon as the electrons originating from a spin singlet state can be coherently separated, they will form an entangled state [8] which gives rise to the possibility of creating and detecting electronic entanglement.

Moving to the detection of entanglement and more involved nanoeletronic devices the interplay of superconductivity with the interactions on a quantum dot become of interest both for phonon [9] and electronic [10] interaction. In this case specifically the interplay of superconductivity with the Kondo effect [11] is of special interest.

Finally the effect of interactions can be large enough to even create a new topological ground state [7] in one-dimensional superconducting structures that may lead to interesting phenomena as emerging Majorana fermions. This paves the way towards electronic quantum computing.

In this review, we will address these different areas in the following order. In Section 2, we will introduce the general concept of superconducting nanoelectronic systems and will then deep-dive into the different subjects mentioned before. In Section 3, we will discuss the effects of interaction when connecting a superconductor to an interacting system. Section 4 will be concerned with the study of Cooper pair splitting and electronic entanglement and we will discuss the emergence and usage of Majorana fermions in superconducting heterostructures in Section 5. We will conclude in Section 6.

\section{General Theory of Transport in Superconducting Heterostructures}

As we want to confine ourselves to the study of classical superconductors, the possibility of creating electron-hole pairs and destroying them from the new ground state gives a finite expectation value to operators of the kind $c_{k \uparrow} c_{-k \downarrow}$, where $c_{k \sigma}$ refers to an annihilation operator for an electron with spin $\sigma$. We will use the mean-field Hamiltonian in the form

$$
H_{S}=\sum_{k \sigma} \varepsilon_{k}\left(c_{k \sigma}^{+} c_{k \sigma}-\Delta \sum_{k} c_{k \uparrow}^{+} c_{-k \downarrow}^{+}+c_{-k \downarrow} c_{k \uparrow}\right) ;
$$

where $\varepsilon_{k}$ describes the kinetic energy of the electrons and the so-called superconductor gap $\Delta$ parametrizes the aforementioned finite expectation value.The reason for the singlet nature of the interaction is the fact that for classical superconductivity phonon-interaction between the electrons is responsible for forming Cooper pairs which is symmetric with respect to the wave vector. This in turn leads to the singlet state being the favored ground state (for a review see [12]). Please mind that we use units such that $e=\hbar=k_{B}=1$.

The resulting wave function of the two electrons can to be written as

$$
|\psi\rangle=\frac{1}{\sqrt{2}}(|\uparrow\rangle|\downarrow\rangle-|\downarrow\rangle|\uparrow\rangle) .
$$


The state in Equation (2) is not entangled as the two electrons are indistinguishable. However, as soon as the electrons are split, the state becomes entangled.

In order to study the separation of electrons the superconductor has to be connected to another system, which is modelled via the introduction of a tunneling Hamiltonian [13]

$$
H_{T 1}=\sum_{\sigma} \gamma_{1}\left[\psi_{1 \sigma}(x=0, t) c_{\sigma}(x=0, t) e^{i \tilde{\varphi} / 2}+H . c .\right] \text {, }
$$

where the operators $\psi_{1 \sigma}$ refer to the electronic annihilation and creation operators in the adjacent material and we have deliberately assumed that tunneling is taking place at $x=0$ (quantum point contact) with a strength that is parametrized by $\gamma_{1}$. A possible phase shift is described by $\tilde{\varphi}$. Scattering phase shifts will naturally disappear from the calculation except for topological cases which are treated in Section 5. The presence of this interface tunneling leads to the fact if a normal metal is connected to a superconductor the superconducting correlations also emerge into the normal metal but decay exponentially with a length scale that is given by the Ginzburg-Landau length [14]. Specifically, when considering also ferromagnetic materials, however, there is no reason to believe that Equation (3) will capture all possible tunnel processes as also spin-dependent scattering potentials may occur giving rise an additional spin-flip contribution to the Hamiltonian [14]

$$
H_{T 2}=\sum_{\sigma} \gamma_{S}\left[\psi_{1 \sigma}(x=0, t) c_{-\sigma}(x=0, t)+H . c .\right] .
$$

The transport between normal metals and superconductors is a well-studied problem [15] [16] [17]. Tunneling of single electrons is only possible for energies larger than the superconducting gap. In an electronic system this energy is either provided by temperature or an external voltage. For energies below the gap electrons that enter the superconductor have to form a Cooper pair which means that they will be back-reflected as a hole. This process is known as Andreev reflection and is suppressed in a typical tunneling environment as it is a secondorder process in tunneling [18]. In a low-temperature environment this allows to use the normal-superconductor junction as a probe for the density of states of a superconductor [12] which can be expressed as a function of energy $\omega$

$$
\rho_{S}=\rho_{0 S}|\omega| / \sqrt{\omega^{2}-\Delta^{2}},
$$

where $\rho_{0 S}$ is a constant reflecting the density of states in the normal conducting case and can be assumed constant in the wide-band limit (no dependency on $\omega)$. As the density of states of a superconductor diverges at the gap-energy of the superconductor $\Delta$, the electrons form a bound state at the interface known as Andreev bound state at $\epsilon= \pm \Delta$.

Studying tunneling between superconductors and ferromagnets Equation (4) becomes important as the spin-active tunneling will also give rise to spin-flip tunneling processes to the ferromagnet as well as the presence of triplet correlations [19] [20]. The different possible charge transport processes are summarized in Figure 1. 
(a)

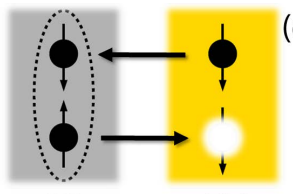

SC
FM

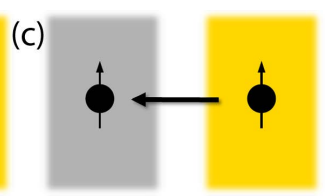

SC
FM

(b)

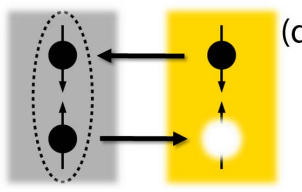

(d)

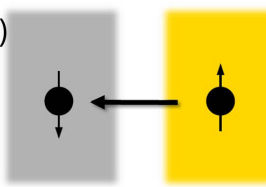

Figure 1. Different processes in a superconductor-ferromagnet quantum point contact with spin-active scattering: in (a) we show the typical Andreev reflection and in (c) we show the typical single-electron transmission between a superconductor and a ferromagnet. In (b) we show the spin-flipped Andreev process involving a spin-flip at the interface and giving rise to triplet correlations in the ferromagnet. Likewise also spin-flip transmissions occur as indicated in (d).

These different tunnel processes emerge naturally in typical tunnel setups as [2], where a superconductor and a ferromagnet are connected on the nanoscale and single electron tunneling, Andreev reflection, spin-flip tunneling and spinflipped Andreev processes can be observed [20].

Tunneling between superconductors is more complicated since Andreev tunneling may happen multiple times before the electron-hole pairs may overcome the barrier of Andreev reflection [21]. This phenomenon of multiple Andreev reflection is well-known and is accompanied by the usual Josephson current which is an $\mathrm{AC}$ voltage whose frequency is determined by the voltage applied across the junction [22] [23] [24].

In the next section we want to deep-dive into the topic of more involved tunneling setups where a quantum dot is positioned between the two leads of the electronic system. In this case the quantum dot provides an additional system to describe that is additionally subjected to electronic and phononic interaction. The study of these effects will be the topic of the next section.

\section{Interactions in Superconductor Heterostructures}

Quantum dots experimentally are small electronic islands. These represent small potential boxes with a large but roughly constant energy spacing of $\delta E$ for the spin-degenerate electronic levels. The capacitance $C$ of such small islands can be very small such that the occupation of both spin levels of the same energy needs the large energy cost for adding one electron (charging energy)

$U=e^{2} / C$. Typically the experimental situation is such that $\delta E>U>k_{B} T, V$. Capacitive coupling of a gate electrode at a certain voltage $V_{g}$ to the quantum dot allows to vary the electronic levels on the quantum dot [12].

The quantum dot in this case can be simplified to the description of just a single electronic level with the additional of the relevant interaction. The quantum dot without interaction in the presence of a magnetic field $B$ is given by

$$
H_{D}=\sum_{\sigma}\left(\varepsilon_{D}+\sigma h / 2\right) d_{\sigma}^{+} d_{\sigma}=: \sum_{\sigma} \epsilon_{D \sigma} d_{\sigma}^{+} d_{\sigma}
$$


where $\varepsilon_{D}$ is the bare quantum dot energy and $h$ refers to the strength of the magnetic field $B$ rescaled in the typical units.

The above model involving a superconducting and a normal conducting lead can be solved exactly [25] [26] [27] [28] and lead to the same transport phenomena as described in Figure 1. In this case, however the transport is related both to the energy-dependence of the density of states of the superconductor as the density of state of the quantum dot which is an electronic level peaked around the bare energy.

Introducing interaction leads to a more complicated setup as we now need to keep track of the repulsive interaction between the electrons of the opposite spin on the quantum level which is described by

$$
H_{D}=U d_{\uparrow}^{+} d_{\uparrow} d_{\downarrow}^{+} d_{\downarrow},
$$

where the interaction is described by $U$ and the local dot electrons are described by annihilation and creation operators $d_{\sigma}^{+}$, where $\sigma$ is referring to the local spin. Likewise we have to consider that the quantum dot may have additional degrees of freedom as vibration which is most prominent when contacting the system to DNA molecules, nanowires of carbon nanotubes. These vibrational degrees of freedom typically have one prominent frequency $\omega_{0}$ and the local phonons are described by bosonic operators $b, b^{+}$

$$
H_{P h}=\omega_{0}\left(b^{+} b+1 / 2\right),
$$

and the interaction is given by

$$
H_{P h}=\sum_{\sigma} d_{\sigma}^{+} d_{\sigma} \lambda_{0}\left(b^{+}+b\right)
$$

The general solution of the system involving interactions is not reached so far but quantitatively good approaches to all situations covered in experiments [29] [30] have been found. We first want to discuss the electronic interaction described in Equation (7).

The non-interacting case is typically not realized but when treating interactions exactly but to lowest order in voltage and for the case with even population of electrons on the quantum dot, it emerges that interaction only leads to a renormalization of the tunneling efficiency [31] so that the non-interacting case with effective tunneling rates is a good approximation for the interacting case with even population.

In the same way the situation of odd occupation can be treated. In this case the quantum dot obtains a localized magnetic moment of spin $1 / 2$. At energy scales below a characteristic temperature $T_{K}$ the Kondo effect comes into play. The electron forms a magnetic impurity that is antiferromagnetically screened leading to the formation of a singlet which is a much more effective scatterer than the impurity itself [32]. The scattering amplitude in this case is strongly increased leading to an increased and even perfect conductance for voltages around the level energy of the impurity which has been observed in many experiments [33].

In the case of a superconductor-quantum dot-normal conductor junction and 
if the Kondo resonance whose resonance width $\Gamma_{K}$ is smaller than the superconducting pairing energy $\Delta$ the Kondo effect on the superconducting side is strongly suppressed which is the usual situation for superconductors [10]. In this case, the Kondo effect can be taken into account by describing the dot-lead transmission as a resonant level with the normal-conductor and the interaction with the superconductor can be treated as a simple tunneling contact (meaning that it will have no energy-dependence). The transmission coefficients of the single-electron tunneling superconducting tunnel contact therefore have to be dressed with the relevant Kondo density of states $\Gamma_{K}^{2} /\left[(\omega-V)^{2}+\Gamma_{K}^{2}\right]$ whereas the Andreev transmission coefficient has to be dressed with $\Gamma_{K}^{4} /\left\{\left[(\omega-V)^{2}+\Gamma_{K}^{2}\right]\left[(\omega+V)^{2}+\Gamma_{K}^{2}\right]\right\}$ in order to properly take holes and elec- trons into account. This treatment has been verified when comparing to experiment [22].

This simple picture, however only holds true as long as long as correlation effects can be neglected. Correlations effects (except for the Kondo effect and superconductivity itself) can typically not be observed from simple measurements of the current or conductance but require more involved measurements of higherorder cumulants like the noise [34].

In this case perturbation theory [35] shows that the interaction of the dot leads to enhanced correlations between the electrons. These correlations emerge as two electrons may transfer to the quantum, dot, and feel their Coulomb repulsion so that they are coherently backreflected to one of the leads attached [36]. In the case of superconductors these correlations may not only lead to enhanced two-particle tunneling but due to Andreev reflection may infer threeand four-particle coherent tunneling terms. As these additional tunneling terms are true effects of interaction they may not be observed in typical limiting cases as the $U \rightarrow \infty$ [37] or $\Delta \rightarrow \infty$ [38] limit.

Finally in such situations also phonon interactions can be taken into account referring to typical situations as e.g. a nanowire being coupled to a normal conductor and a superconductor as shown in Figure 2.

The treatment of superconducting systems including phonon-interaction is particularly cumbersome due to the various energy-scales which are present in the problem [39] [40] [41] [42].

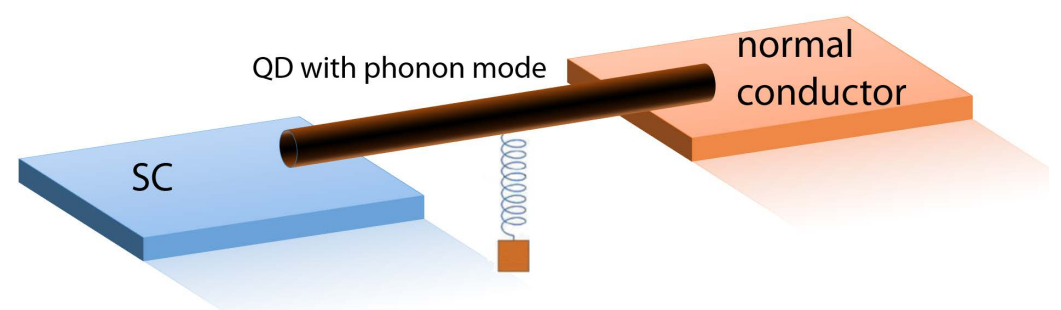

Figure 2. Sketch of the system: a quantum dot with strong onsite Coulomb interaction is assumed to be in the Kondo regime and its resonance width is given by $\Gamma_{K}$. It is coupled to a normal conductor and a superconductor. The quantum dot is subjected to a finite bias voltage and coupled to a phonon mode. 
In the case of a quantum dot in the Kondo regime which is additionally affected by phonon-interaction the most prominent effect is the emergence of much more prominent Andreev conductance steps due to the additional interaction with the phonons [39].

The interaction effects which have been discussed before can not only be observed in the typical normal conductor-superconductor heterostructures but also in ferromagnet-superconductor heterostructures [6]. In these heterostructures we typically see the different processes that we have indicated in Figure 1 [20].

The most prominent feature in these setups is the fact that in the case of a quantum dot in the Kondo regime not just a single Kondo peak is observed but two. This feature can be attributed to the fact that the the two states withn spinup and spin-down are split with respect to the exchange field of the ferromagnet. This exchange-field induced splitting of the resonance peaks is a very effective spin-filter in such setups as just by tuning the voltage the preferred spin-direction can be chosen. Indeed, even for typical polarisations of the ferromagnet as $46 \%$ is has been experimentally shown that spin polarisations of the current of up to $70 \%$ can be reached (see Figure 3 ).

This spin-filtering mechanism is extremely relevant for the discovery of entanglement in superconducting heterostructures which will be the topic of the next section.

\section{Cooper Pair Splitters}

In Equation (2), we have mentioned the possbility of generating entangled electron pairs by splitting the two electrons of a Cooper pair. Indeed this feature has been identified as a possible source of electronic entanglement early-on [43]. However, the actual recipe for performing high-efficiency Cooper pair splitting has been a focal point of research [30].

A basic splitting device is a three-terminal system with a central superconductor and two normal terminals which are attached to the device. If the electrons can be split as in Figure 4(a) the pair represents an entangled electron pair. However, as soon as a typical splitting device is operated at finite bias the process in (a) has

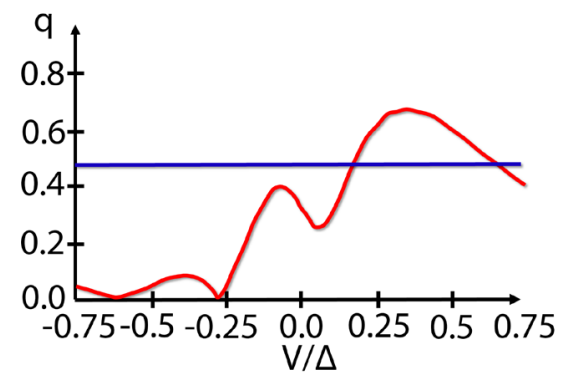

Figure 3. Calculation of the quality factor of spin-filtering $q$ (ratio of the conductances per spin direction over the the sum of the conductances) with using the experimental parameters as in [6]. Andreev reflections have been neglected since under these conditions they do not contribute strongly. The quality factor reaches about $70 \%$ even at finite temperature as in the experiment. The blue line indicates the quality factor of a simple tunneling junction to a ferromagnet with equal polarisation. 

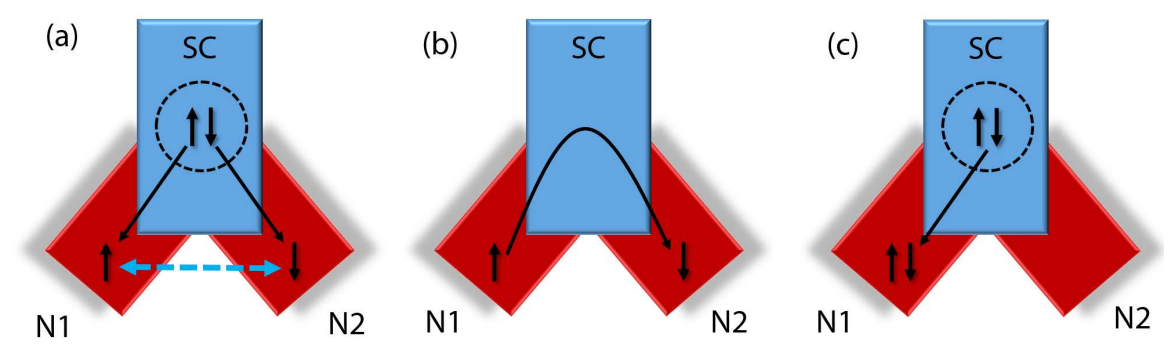

Figure 4. (a) Illustration of Cooper pair splitting in a Y-junction geometry with the superconductor. The superconductor acts as a source of entangled electron pairs in the normal drains. (b): Process of elastic cotunneling that becomes possible at finite bias between the normal leads. (c) Local Andreev reflections transfer pairs of electrons to the same lead.

to compete with the process of elastic cotunneling (b) and local Andreev reflection (c).

Under adequately chosen experimental conditions the process of Cooper pair splitting can indeed be observed also in simple structures connecting a superconductor to two normal conductors [26] but the efficiency of this process is limited due to the limited number of scalable parameters in this setup. When connecting the superconductor to two ferromagnets the possibility of spin-flipping at the interface also allows observing entangled pairs of electrons with equal spin (p-wave splitting) as e.g.

$$
\left|\Phi^{ \pm}\right\rangle=\frac{1}{\sqrt{2}}\left(|\uparrow\rangle_{A}|\uparrow\rangle_{B} \pm|\downarrow\rangle_{A}|\downarrow\rangle_{B}\right) .
$$

The different charge transfer processes in this case are also summarized in the picture below. (Figure 5)

As discussed above superconductor-ferromagnet interfaces inherently allow for spin-active scattering at the interface so that ferromagnet-superconductor hybrid structures will allow to generate split $p$-wave Cooper pairs even if no $p$-wave superconductor is present. This process has been referred to as spinflipped crossed Andreev reflection [26].

In addition the possbility of Andreev reflection between the superconductors allows for another process driving positive correlations. In case of a voltage close to the superconductor gap being applied across the two normal conductors the dominant charge transfer process becomes an Andreev reflection with one normal conductor and as consecutive Andreev reflection to the second normal conductor via the superconductor. This is the process of Andreev reflection enhanced transmission [25].

However, splitters realized using just a single quantum dot do have a limited set of parameters to really allow for high effiency splitting. In thie case one has to go over to a double-dot setup as depicted in Figure 6.

Cooper pair splitters can also be analyzed in the general interacting case. In this case the general Hamiltonian for a double quantum dot system with a superconducting leads and onsite interaction needs to be applied following Equation (6) and Equation (7). The system can be solved exactly in the non-interacting 

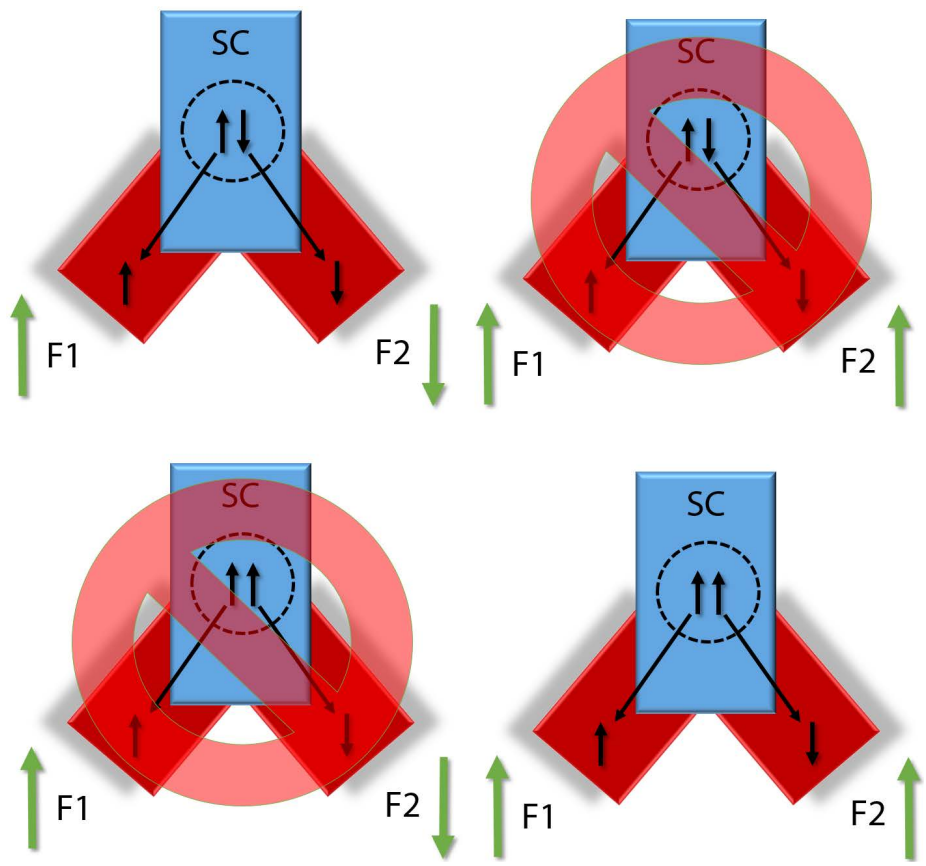

Figure 5. Summary of the possible charge transfer processes in a superconductor ferromagnet device. The superconductor (blue) and the ferromagnets (red, are assumed to be fully polarized) are coupled via a quantum dot. The polarisation is indicated by green arrows. In the upper part, the situation for a s-wave SC is shown. The Cooper pair may split if the two FMs are antiparallely polarized-In the lower part the situation for spinpolarized p-wave SCs is depicted. The Cooper pair may now only split if the two FMs are equally polarized.

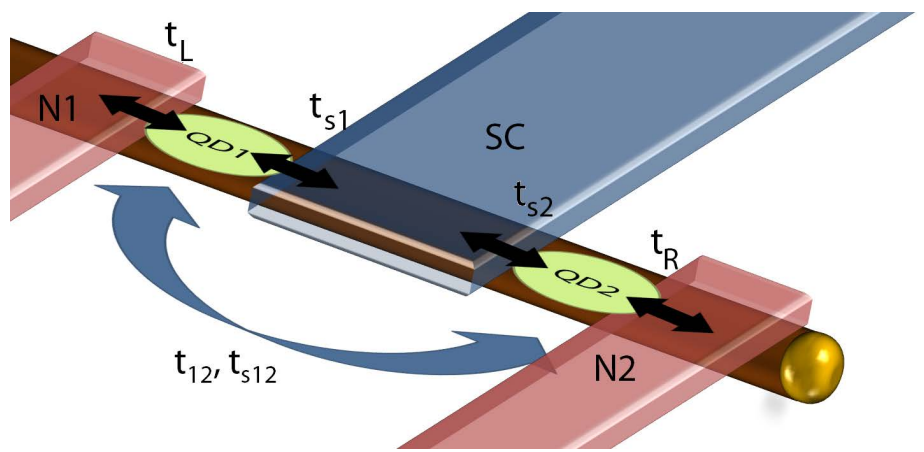

Figure 6. Schematics of the generic model for a typical $Y$-junction: a SC is connected via two quantum dots to two normal leads possibly at a different chemical potential. The two quantum dots have tunable levels and may be coupled either via a the superconductor or directly.

case. The onsite Coulomb-interaction can be approximately dealt with assuming that on-resonance the interaction only leads to a renormalization of the coupling as described above [44].

Indeed such an approach allows for a description of the experimental results in the case of carbon nanotubes and InAs nanowires. The key differences between the two setups can also be traced back to the material properties resulting in InAs nanowires having a Cooper-pair splitting transmission amplitude with a specific dependency on the spatial separation of the two leads and 
the superconducting density of states [31] whereas the transmission amplitude in case of carbon nanotubes is just constant [45].

This general solution to Cooper pait splitters also allows to move forward with the real problem at hand which is to show that the generated electron pairs are indeed entangled. Entanglement can be proven using the Bell parameter in the typical CHSH inequality [46]

$$
\varepsilon=\left|E\left(\boldsymbol{m}_{A}, \boldsymbol{m}_{B}\right)+E\left(\boldsymbol{m}_{A}^{\prime}, \boldsymbol{m}_{B}\right)+E\left(\boldsymbol{m}_{A} \boldsymbol{m}_{B}^{\prime}\right)-E\left(\boldsymbol{m}_{A}^{\prime}, \boldsymbol{m}_{B}^{\prime}\right)\right|,
$$

where the correlator is given by $E\left(\boldsymbol{m}, \boldsymbol{m}^{\prime}\right)=P_{m m^{\prime},+}+P_{m m^{\prime},--}-P_{m m^{\prime},-+}-P_{m m^{\prime},+-}$ denotes the probability of observing an electron pair in detectors with directions $\boldsymbol{m}, \boldsymbol{m}^{\prime}$ with spin detectors $\sigma= \pm, \sigma^{\prime}= \pm$. In our case ferromagnetic leads act as spin detectors with a certain polarisation or efficiency $P$, which is typically quite limited on the nanoscale.

In order to obtain the probabilities of certain two-particle processes from the conductances in a typical experiment we can use the general relation

$$
P_{i j}=\frac{G_{i j}}{\sum G_{k l}}
$$

where $P_{i j}$ refers to the probability of the process involving the specifics of the electrons $i$ and $j$ and we have measured the corresponding conductances. Since we can immediately deduct from our analysis that the probabilities do only depend on the pairwise alignment of the superconductors the measurements can also be performed in the typical Cooper pair splitter geometry (see Figure 7).

Even more, one can show that the conductance has a characteristic form (independent of the specifics of the Cooper pair splitter)

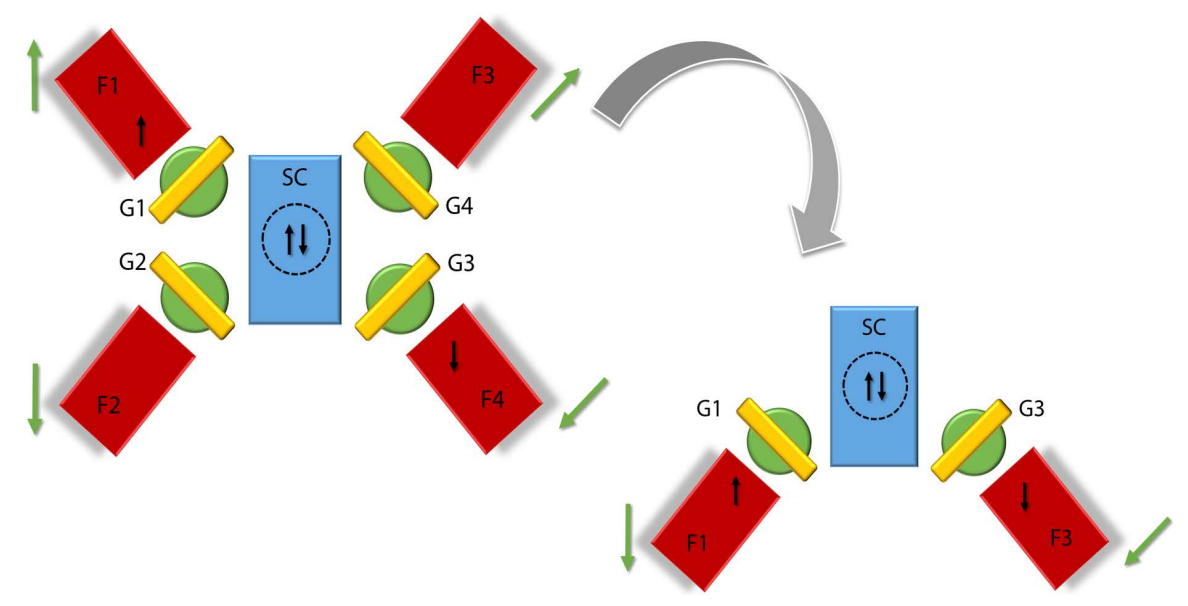

Figure 7. Sketch of the experimental setups. (a) A central superconducting finger $S$ (blue) is contacted to two InAs nanowires (brown). These in turn are contacted by four ferromagnetic electrodes (brown). These in turn are contacted by four ferromagnetic electrodes (red) and the direction of magentization of which are indicated by arrows here for the configuration of the magnetization directions. Alice and Bob are represented by the spin detectors consisting of electrodes F1, F2 and F3, F4 respectively. The emerging quantum dots between the superconducting finger and the four ferromagnetic electrodes are tunable by top gates G1-G4. (b) Using our analysis the setup can be considerably simplified to a $Y$-junction geometry. 


$$
G_{i j}=f(V, \delta r, \Sigma)\left(1-\boldsymbol{g}_{i} \boldsymbol{g}_{j}\right),
$$

With the $f(V, \delta r, \Sigma)$ a function of the applied bias voltage $V$, the width $\delta r$ of the superconductor and a possible on-site interaction on the quantum dot described by a self-energy $\Sigma$ as well as the magnetisation vectors of the ferromagnets described by unit vectors in the direction of magnetisation and their polarisation $P$. This function drops out of the calculation once we calculate the actual Bell parameter

$$
\in=P^{2} \epsilon_{0}, \in_{0}=\left|\overrightarrow{\boldsymbol{m}_{A} \boldsymbol{m}_{B}+\boldsymbol{m}_{A}^{\prime} \boldsymbol{m}_{B}+\boldsymbol{m}_{A} \boldsymbol{m}_{B}^{\prime}-\boldsymbol{m}_{A}^{\prime} \boldsymbol{m}_{B}^{\prime}}\right|
$$

Therefore one can measure the violation of Bell's inequality also from a typical Cooper pair splitter setup. However, the interesting question is whether we will be able to prove that entanglement exists as typical polarisations are only of the order of $40 \%$. Given that the maximal value of $\epsilon_{0}$ is $2 \sqrt{2}$ polarisations required to violate Bell's inequality are typically not within reach.

However, we can still overcome this by using two effects. First, we have shown already that operating the ferromagnet-quantum-dot junction in the Kondo limit we can reach effective polarisations of $\sim 70 \%$. Furthermore, we do not have to violate Bell's inequality to prove the existence of entanglement. The maximal value of $\epsilon_{0}$ for a separable quantum state is only $\sqrt{2}$ and therefore we only need to reach

$$
P^{2} 2 \sqrt{2} \geq \sqrt{2} \rightarrow P^{2} \approx 70 \%
$$

This means that proving the generation of entanglement should be possible within the usual beamsplitter setups and would represent the natural next step.

\section{Majorana Fermions in Superconducting Heterostructures}

With this we want to move away from interactions on a quantum dot and approach interactions in one-dimensional structures. It is well-known that $p$ wave superconductors with a single spin species have two different topological phases that are illustrated in Figure 8.

In the case of two single electrons at the ends these electons are still part of the condensate of Cooper pairs so that they represent zero-energy bound states. As they have zero energy this makes them fermions which are their own antiparticle and therefore Majorana fermions.

The interesting question which emerges is whether the situation of single
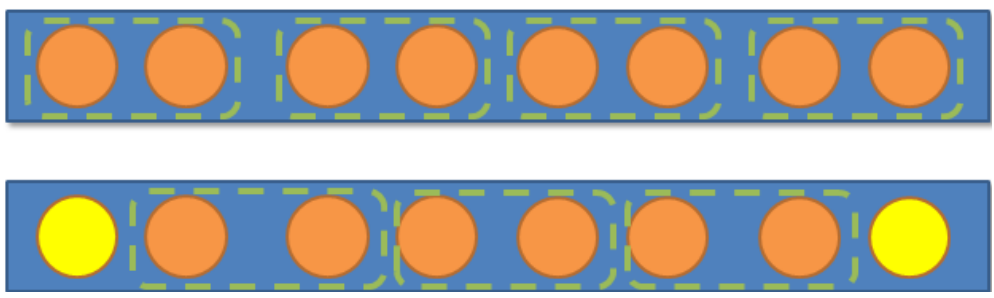

Figure 8. Sketch of the two topological phases of a p-wave superconductor. In the first phase all electrons are paired in Cooper pairs. In the second phase one pair is broken and single electrons appear at the end of the superconductor. 
electrons at the ends can actually be realized as a stable state in reality and indeed this has been recently shown in [7].

Let us assume that we have a quantum wire on top of a spin-polarized $p$-wave superconductor. The superconductor would induce $p$-wave correlations in the quantum wire described by an order parameter $\Delta_{p}$ so that the tight-binding Hamiltonian for the wire at finite length at chemical potential $\mu_{\text {wire }}$ can be written as

$$
H=-\mu_{\text {wire }} \sum_{x=1}^{N} c_{x}^{+} c_{x}+\sum_{x=1}^{N-1}\left(-t_{\text {wire }} c_{x}^{+} c_{x+1}+\Delta_{p} c_{x} c_{x+1}+H . c .\right)
$$

where $t_{\text {wire }}$ refers to the local tunneling strength. For simplicity let us assume $\mu_{\text {wire }}=0, t_{\text {wire }}=\mu_{\text {wire }}$, in which we can write

$$
H=\sum_{x=1}^{N-1}\left(-t_{\text {wire }} c_{x}^{+} c_{x+1}+t_{\text {wire }} c_{x} c_{x+1}+H . c .\right)
$$

Here the fermionic operators can be written in terms of Majorana operators $\gamma_{\alpha, x}=\gamma_{\alpha, x}^{+}, \alpha=A, B$ via

$$
c_{x}=\frac{1}{2}\left(\gamma_{B, x}+i \gamma_{A, X}\right), c_{x}^{+}=\frac{1}{2}\left(\gamma_{B, X}-i \gamma_{A, X}\right)
$$

Then from Equation (17) we find

$$
H=-i t_{\text {wire }} \sum_{x=1}^{N-1} \gamma_{B, x} \gamma_{A, x+1}
$$

Which is a simple tight-binding Hamiltonian for Majorana fermions. A similar mapping can be achieved at $\Delta_{p} \neq t_{\text {wire }}$, which is, however, more complicated. Two of the Majorana fermions always combine into one ordinary fermion $d_{x}=\frac{1}{2}\left(\gamma_{A, x+1}+\gamma_{B, x}\right)$. The formation of a fermion costs $2 \Delta_{p}=2 t_{\text {wire }}$ of energy due to the presence of the superconductor so that the Majorana fermion picture survives up to $\left|\mu_{\text {wire }}\right|=2 t_{\text {wire }}$.

As discussed above, two Majorana fermions will remain uncoupled $\gamma_{A, 1}, \gamma_{B, N}$. These two are therefore the uncoupled Majorana fermions we were looking for.

From here two directions of research have emerged. One is focussed on the detection of Majorana fermions and the other is focussed on the generation of an appropriate situation for the emergence of a Majorana fermion.

For the former line of research the most apparent feature is perfect transmission through a Majorana fermion at 0 voltage. This phenomenon is related to a scattering phase shift that is undergone during Andreev reflection from the superconductor hosting a Majorana fermion. Equation (3) acquires a phase shifft $\tilde{\varphi}= \pm \varphi$ for electrons/ holes. In the topological phase the shift is $\varphi=\pi$ [47]. In this case the Andreev bound states at $\epsilon= \pm \Delta$ merge into one bound state at

$$
\varepsilon= \pm \Delta \cos (\varphi / 2)= \pm \Delta \cos (\pi / 2)=0
$$

The emergence of this bound state is the reason for perfect conductance through a Majorana fermion at zero voltage [48]. Additionally the phase shift can be recognised in a characteristic noise behavior of Majorana devices. 
Second, research has focussed on creating systems that mimic the behavior of the $p$-wave superconductor setup described above. This mimicking includes several possible suggestions of systems which all need to have both a $p$-wave gap as well as a superconduting gap with the $p$-wave gap dominating the behavior. Identified candidates of this kind are strongly spin-orbit coupled quantum wires proximity coupled to superconductors in a magnetic field [49] [50], exciton condensates proximity coupled to $p$-wave superconductors [51] and ferromagentic wires proximity coupled to superconductors [52]. The analysis of these system thereby always proceeds via denoting the full Hamiltonian and identifying nontrivial bound states of the system that could represent Majorana fermions.

The beauty of Majorana fermions fundamentally lies in their applicability for quantum computing as their state is topologically protected (due to the necessary shift in their topological state) [53]. As long as they are not contacted by the outside world the different quantum states they can be in

- Both are electrons.

- Left hole, right electron.

- Left electron, right hole.

- Both are holes.

Can be protected and read out by connecting them to an electronic lead [54]. However, also the interactions with an external environment can be exploited allowing for efficient preparation of an initial state of the Majorana fermion system [54].

\section{Conclusions}

To conclude we have reviewed several aspects of the latest research on superconductor hybrids including the setup of superconducting point contact as well as more complicated structures. This has been accompanied by studying interactions in such systems as well as the possible generation of entanglement. Finally, we have reviewed the latest results for generation and detection of Majorana fermions.

In conclusion, superconductor hybrids still provide a very relevant and interesting field of research specifically for the study of electronic entanglement. They pave the way towards truly electronic quantum computing and offer a wide variety of electronic building blocks to allow for implementation of more complicated electronic devices.

However, the general drawback of necessarily low temperatures remains which also demonstrates the need for finding technologically appropriate solutions including exciton condensates [55] [56].

\section{Acknowledgements}

The author would like to thank I. Deppner for numerous discussions.

\section{References}

[1] Onnes, H.K. (1911) The Superconductivity of Mercury. Comm. Phys. Lab. Univ., Leiden, 122-124. 
Breyel, D., Schmidt, T.L. and Komnik, A. (2012) Rydberg Crystallization Detection by Statistical Means. Physical Review A, 86, Article ID: 023405. https://doi.org/10.1103/physreva.86.023405

[2] Bardeen, J., Cooper, L.N. and Schrieffer, J.R. (1957) Theory of Superconductivity. Physical Review, 108, 1175-1204. https://doi.org/10.1103/PhysRev.108.1175

[3] Tinkham, M. (1996) Introduction to Superconductivity. Courier Corporation, North Chelmsford.

[4] De Franceschi, S., Kouwenhoven, L., Schönenberger, C. and Wernsdorfer, W. (2010) Hybrid Superconductor-Quantum Dot Devices. Nature Nanotechnology, 5, 703711. https://doi.org/10.1038/nnano.2010.173

[5] Mourik, V., Zuo, K., Frolov, S.M., Plissard, S.R., Bakkers, E.P.A.M. and Kouwenhoven, L.P. (2012) Signatures of Majorana Fermions in Hybrid Superconductor-Semiconductor Nanowire Devices. Science, 336, 1003-1007. https://doi.org/10.1126/science. 1222360

[6] Hofstetter, L., Geresdi, A., Aagesen, M., Nygård, J., Schönenberger, C. and Csonka, S. (2010) Ferromagnetic Proximity Effect in a Ferromagnet-Quantum-Dot-Superconductor Device. Physical Review Letters, 104, Article ID: 246804. https://doi.org/10.1103/PhysRevLett.104.246804

[7] Kitaev, A.Y. (2001) Unpaired Majorana Fermions in Quantum Wires. Physics-Uspekhi, 44, 131-136. https://doi.org/10.1070/1063-7869/44/10S/S29

[8] Recher, P. and Loss, D. (2002) Creation of Nonlocal Spin-Entangled Electrons via Andreev Tunneling, Coulomb Blockade, and Resonant Transport. Journal of Superconductivity, 15, 49-65.

[9] Sköldberg, J., Löfwander, T., Shumeiko, V.S. and Fogelström, M. (2008) Spectrum of Andreev Bound States in a Molecule Embedded inside a Microwave-Excited Superconducting Junction. Physical Review Letters, 101, Article ID: 087002.

[10] Nussbaumer, T. and Belzig, W. (2004) Quantum Dot Coupled to a Normal and a Superconducting Lead. Nanotechnology, 15, S479-S482. https://doi.org/10.1088/0957-4484/15/7/056

[11] Kondo, J. (1964) Resistance Minimum in Dilute Magnetic Alloys. Progress of Theoretical Physics, 32, 37-49. https://doi.org/10.1143/PTP.32.37

[12] Soller, H. (2009) FCS for Superconducting Quantum Point Contacts. Doctoral Dissertation.

[13] Cohen, M.H., Falicov, L.M. and Phillips, J.C. (1962) Superconductive Tunneling. Physical Review Letters, 8, 316-318. https://doi.org/10.1103/PhysRevLett.8.316

[14] Yamada, Y., Tanaka, Y. and Kawakami, N. (2011) Interplay of Kondo and Superconducting Correlations in the Nonequilibrium Andreev Transport through a Quantum Dot. Physical Review B, 84, Article ID: 075484. https://doi.org/10.1103/PhysRevB.84.075484

[15] Soller, H. (2014) Proximity Effect in Normal-Superconductor Hybrids at the Nanoscale. Journal of Applied Mathematics and Physics, 2, 745-752. https://doi.org/10.4236/jamp.2014.28082

[16] Muzykantskii, B.A. and Khmelnitskii, D.E. (1994) Quantum Shot Noise in a Normal-Metal-Superconductor Point Contact. Physical Review B, 50, 3982-3987. https://doi.org/10.1103/PhysRevB.50.3982

[17] Cuevas, J.C., Martin-Rodero, A. and Yeyati, A.L. (1996) Hamiltonian Approach to the Transport Properties of Superconducting Quantum Point Contacts. Physical Review B, 54, 7366-7379. https://doi.org/10.1103/PhysRevB.54.7366

[18] Andreev, A.F. (1964) Thermal Conductivity of the Intermediate State of Supercon- 
ductors. JETP Letters, 46, 1823-1828.

[19] Grein, R., Löfwander, T., Metalidis, G. and Eschrig, M. (2010) Theory of Superconductor-Ferromagnet Point-Contact Spectra: The Case of Strong Spin Polarization. Physical Review B, 81, Article ID: 094508.

[20] Soller, H., Hofstetter, L., Csonka, S., Yeyati, A. L., Schönenberger, C. and Komnik, A. (2012) Kondo Effect and Spin-Active Scattering in Ferromagnet-Superconductor Junctions. Physical Review B, 85, Article ID: 174512. https://doi.org/10.1103/physrevb.85.174512

[21] Pérez-Willard, F., Cuevas, J.C., Sürgers, C., Pfundstein, P., Kopu, J., Eschrig, M. and Löhneysen, H.V. (2004) Determining the Current Polarization in Al/Co Nanostructured Point Contacts. Physical Review B, 69, Article ID: 140502. https://doi.org/10.1103/PhysRevB.69.140502

[22] Soller, H. (2013) Fcs of Superconducting Tunnel Junctions in Nonequilibrium. International Journal of Modern Physics B, 27, Article ID: 1350072. https://doi.org/10.1142/s0217979213500720

[23] Soller, H. and Komnik, A. (2011) Hamiltonian Approach to the Charge Transfer Statistics of Kondo Quantum Dots Contacted by a Normal Metal and a Superconductor. Physica E: Low-Dimensional Systems and Nanostructures, 44, 425-429. https://doi.org/10.1016/j.physe.2011.09.014

[24] Cuevas, J.C., Martín-Rodero, A. and Yeyati, A.L. (1999) Shot Noise and Coherent Multiple Charge Transfer in Superconducting Quantum Point Contacts. Physical Review Letters, 82, 4086-4089. https://doi.org/10.1103/PhysRevLett.82.4086

[25] Soller, H. and Komnik, A. (2011) Charge Transfer Statistics and Entanglement in Normal-Quantum Dot-Superconductor Hybrid Structures. The European Physical Journal D, 63, 3-8. https://doi.org/10.1140/epjd/e2010-00256-7

[26] Soller, H. and Komnik, A. (2012) P-Wave Cooper Pair Splitting. Beilstein Journal of Nanotechnology, 3, 493-500. https://doi.org/10.3762/bjnano.3.56

[27] Machon, P., Eschrig, M. and Belzig, W. (2013) Nonlocal Thermoelectric Effects and Nonlocal Onsager Relations in a Three-Terminal Proximity-Coupled Superconductor-Ferromagnet Device. Physical Review Letters, 110, Article ID: 047002. https://doi.org/10.1103/PhysRevLett.110.047002

[28] Belzig, W., Wilhelm, F.K., Bruder, C., Schön, G. and Zaikin, A.D. (1999) Quasiclassical Green's Function Approach to Mesoscopic Superconductivity. Superlattices and Microstructures, 25, 1251-1288.

[29] Hofstetter, L., Csonka, S., Baumgartner, A., Fülöp, G., d’Hollosy, S., Nygård, J. and Schönenberger, C. (2011) Finite-Bias Cooper Pair Splitting. Physical Review Letters, 107, Article ID: 136801. https://doi.org/10.1103/physrevlett.107.136801

[30] Schindele, J., Baumgartner, A. and Schönenberger, C. (2012) Near-Unity Cooper Pair Splitting Efficiency. Physical Review Letters, 109, Article ID: 157002. https://doi.org/10.1103/physrevlett.109.157002

[31] Soller, H. (2014) Generic Model for Cooper Pair Splitting. International Journal of Modern Physics B, 28, Article ID: 1450137. https://doi.org/10.1142/s0217979214501379

[32] Soller, H. (2013) Non-Linear Transport Properties of Hybrid Nanoelectronic Devices. Logos Verlag, Berlin.

[33] Goldhaber-Gordon, D., Shtrikman, H., Mahalu, D., Abusch-Magder, D., Meirav, U. and Kastner, M.A. (1998) Kondo Effect in a Single-Electron Transistor. Nature, 391, 156-159. https://doi.org/10.1038/34373

[34] Jehl, X., Sanquer, M., Calemczuk, R. and Mailly, D. (2000) Detection of Doubled 
Shot Noise in Short Normal-Metal/Superconductor Junctions. Nature, 405, 50-53. https://doi.org/10.1038/35011012

[35] Soller, H. and Komnik, A. (2014) Full Counting Statistics of Interacting Quantum Dots Contacted by a Normal Metal and a Superconductor. Europhysics Letters, 106, 37009. https://doi.org/10.1209/0295-5075/106/37009

[36] Schmidt, T.L., Komnik, A. and Gogolin, A.O. (2007) Hanbury Brown-Twiss Correlations and Noise in the Charge Transfer Statistics through a Multiterminal Kondo Dot. Physical Review Letters, 98, Article ID: 056603. https://doi.org/10.1103/PhysRevLett.98.056603

[37] Fazio, R. and Raimondi, R. (1998) Resonant Andreev Tunneling in Strongly Interacting Quantum Dots. Physical Review Letters, 80, 2913-2916.

https://doi.org/10.1103/PhysRevLett.80.2913

[38] Braggio, A., Governale, M., Pala, M.G. and König, J. (2011) Superconducting Proximity Effect in Interacting Quantum Dots Revealed by Shot Noise. Solid State Communications, 151, 155-158. https://doi.org/10.1016/j.ssc.2010.10.043

[39] Albrecht, K.F., Soller, H., Mühlbacher, L. and Komnik, A. (2013) Transient Dynamics and Steady State Behavior of the Anderson-Holstein Model with a Superconducting Lead. Physica E: Low-Dimensional Systems and Nanostructures, 54, 15-23. https://doi.org/10.1016/j.physe.2013.05.019

[40] Zazunov, A., Egger, R., Mora, C. and Martin, T. (2006) Superconducting Transport through a Vibrating Molecule. Physical Review B, 73, Article ID: 214501. https://doi.org/10.1103/physrevb.73.214501

[41] Bai, L., Zhang, Z.Z. and Jiang, L. (2011) Andreev Reflection Current through a Molecule Quantum Dot in the Presence of the Electron-Phonon Interaction and the Spin-Flip Scattering. Physics Letters A, 375, 661-665. https://doi.org/10.1016/j.physleta.2010.11.042

[42] Zazunov, A. and Egger, R. (2010) Adiabatic Polaron Dynamics and Josephson Effect in a Superconducting Molecular Quantum Dot. Physical Review B, 81, Article ID: 104508. https://doi.org/10.1103/physrevb.81.104508

[43] Recher, P., Sukhorukov, E.V. and Loss, D. (2001) Andreev Tunneling, Coulomb Blockade, and Resonant Transport of Nonlocal Spin-Entangled Electrons. Physical Review B, 63, Article ID: 165314. https://doi.org/10.1103/physrevb.63.165314

[44] Chevallier, D., Rech, J., Jonckheere, T. and Martin, T. (2011) Current and Noise Correlations in a Double-Dot Cooper-Pair Beam Splitter. Physical Review B, 83, Article ID: 125421. https://doi.org/10.1103/physrevb.83.125421

[45] Burset, P., Herrera, W.J. and Yeyati, A.L. (2011) Microscopic Theory of Cooper Pair Beam Splitters Based on Carbon Nanotubes. Physical Review B, 84, Article ID: 115448. https://doi.org/10.1103/physrevb.84.115448

[46] Di Lorenzo, A. and Nazarov, Y.V. (2005) Full Counting Statistics with Spin-Sensitive Detectors Reveals Spin Singlets. Physical Review Letters, 94, Article ID: 210601. https://doi.org/10.1103/physrevlett.94.210601

[47] Akhmerov, A.R., et al. (2011) Quantized Conductance at the Majorana Phase Transition in a Disordered Superconducting Wire. Physical Review Letters, 106, Article ID: 057001. https://doi.org/10.1103/PhysRevLett.106.057001

[48] Liu, J., Potter, A.C., Law, K.T. and Lee, P.A. (2012) Zero-Bias Peaks in the Tunneling Conductance of Spin-Orbit-Coupled Superconducting Wires with and without Majorana End-States. Physical Review Letters, 109, Article ID: 267002.

[49] Oreg, Y., Refael, G. and von Oppen, F. (2010) Helical Liquids and Majorana Bound States in Quantum Wires. Physical Review Letters, 105, Article ID: 177002. 
https://doi.org/10.1103/physrevlett.105.177002

[50] Gangadharaiah, S., Braunecker, B., Simon, P. and Loss, D. (2011) Majorana Edge States in Interacting One-Dimensional Systems. Physical Review Letters, 107, Article ID: 036801. https://doi.org/10.1103/physrevlett.107.036801

[51] Soller, H. and Breyel, D. (2013) Signatures in the Conductance for Phase Transitions in Excitonic Systems. Modern Physics Letters B, 27, Article ID: 1350185. https://doi.org/10.1142/S0217984913501856

[52] Soller, H. and Komnik, A. (2014) Charge Transfer Statistics of Transport through Majorana Bound States. Physica E: Low-Dimensional Systems and Nanostructures, 63, 99-104. https://doi.org/10.1016/j.physe.2014.05.020

[53] Beenakker, C.W.J. (2013) Search for Majorana Fermions in Superconductors. Annual Review of Condensed Matter Physics, 4, 113-136. https://doi.org/10.1146/annurev-conmatphys-030212-184337

[54] Soller, H. (2016) Dissipative Quantum Computing with Majorana Fermions. Journal of Applied Mathematics and Physics, 4, 227-232. https://doi.org/10.4236/jamp.2016.42030

[55] Soller, H., Dolcini, F. and Komnik, A. (2012) Nanotransformation and Current Fluctuations in Exciton Condensate Junctions. Physical Review Letters, 108, Article ID: 156401. https://doi.org/10.1103/physrevlett.108.156401

[56] Soller, H. (2015) Exciton Condensates and Superconductors-Technical Differences and Physical Similarities. Journal of Applied Mathematics and Physics, 3, 12181225. https://doi.org/10.4236/jamp.2015.39149

Submit or recommend next manuscript to SCIRP and we will provide best service for you:

Accepting pre-submission inquiries through Email, Facebook, LinkedIn, Twitter, etc. A wide selection of journals (inclusive of 9 subjects, more than 200 journals) Providing 24-hour high-quality service User-friendly online submission system Fair and swift peer-review system Efficient typesetting and proofreading procedure Display of the result of downloads and visits, as well as the number of cited articles Maximum dissemination of your research work

Submit your manuscript at: http://papersubmission.scirp.org/

Or contact jamp@scirp.org 